\title{
Updated Treatment for Acne: Targeted Therapy Based on Pathogenesis
}

\author{
Ichiro Kurokawa (D) A Alison M. Layton · Rei Ogawa
}

Received: March 2, 2021 / Published online: June 11, 2021

(C) The Author(s) 2021 is primarily a disease associated with sebogenesis. Monosaturated free acids are important components. Insulin growth factor 1 (IGF-1) promotes the proliferation and differentiation of sebocytes and IL-1 $\beta$. Research into the microbiome may also provide insights into potential future therapeutic options for acne. Scars, both atrophic and hypertrophic, are common sequelae to acne. Risk factors associated with the development of acne scars include genetic, systemic, local, and lifestyle factors. Pro-inflammatory cytokines have been shown to play a crucial role in the development of acne-induced hypertrophic scars. Treatment for extensive inflammatory keloid scarring is limited. Surgery and postoperative radiotherapy are two possible options. Transforming growth factor- $\beta$ (TGF $\beta$ ), IL-6, matrix metalloproteinase (MMP), IGF-1, and B cells are found in keloid or hypertrophic scar tissues. Biological antibodies targeting these cytokines may be a potential strategy for the prevention and treatment of this type of scar in the future. Future treatment for acne should embrace approaches that target the main etiological factors of acne. In particular, specific emphasis on aggressive treatment in the acute inflammatory phase to reduce the likelihood of scarring and other clinical sequelae, such as pigmentary changes would be highly desirable. Treatment for established acne-induced sequelae should also be considered.
R. Ogawa

Department of Plastic, Reconstructive and Aesthetic Surgery, Nippon Medical School, Tokyo, Japan 
Keywords: Acne; Targeted therapy; Immunology; Microbiome; Biologics; Wound healing; Trifarotene; Cascoterone

\section{Key Summary Points}

Recent advances have facilitated potential novel approaches to acne management owing to improved understanding of the pathophysiology and immunological aspects

Biological antibodies targeting IL-1 $\beta$, IL17, IL-23, and TNF $\alpha$ could provide novel approaches for treating severe acne and related disorders

Research into the microbiome may also provide insights into potential future therapeutic options for acne

Biological antibodies targeting TGF $\beta$, IL-6, MMP, IGF-1, and B cells may be a potential strategy for the prevention and treatment of this type of scar in the future

Future treatment for acne should embrace approaches that target the main etiological factors of acne

\section{DIGITAL FEATURES}

This article is published with digital features, including a summary slide to facilitate understanding of the article. To view digital features for this article go to https://doi.org/10.6084/ m9.figshare.14560572.

\section{INTRODUCTION}

Recent advances have further elucidated the pathogenesis of acne: it is now clear that immunological factors play an important role [1]. To date, acne pathogenesis has implicated four major factors: androgen-dependent sebogenesis, hyperkeratinization of the infundibulum, Cutibacterium acnes (C. acnes) colonization, and inflammation [2]. Successful targeted therapy for acne currently includes topical retinoids that normalize abnormal hyperkeratinization in the infundibulum and novel topical retinoids with anti-inflammatory properties [3]. Topical and oral antimicrobials inhibit bacterial proliferation and reduce inflammation related to cytokines and extracellular enzymes [4]. Topical benzoyl peroxide (BPO) is highly effective in reducing both sensitive and resistant strains of C. acnes and has some impact on hyperkeratinization in the infundibulum $[5,6]$. Anti-androgens can regulate androgen metabolism, resulting in suppression of sebum excretion [7]. Orally administered isotretinoin is currently the only agent that can affect all four main factors implicated in acne [2,3].

This review aims to clarify targets based on acne pathogenesis and summarize treatment options for acne in the future.

This article is based on previously conducted studies and does not contain any new studies with human participants or animals performed by any of the authors.

\section{HOW CAN RECENT ADVANCES IN ACNE PATHOGENESIS INFORM FUTURE TREATMENT?}

Novel understanding of acne pathogenesis may inform future approaches to therapy. Recent updated knowledge of acne pathogenesis includes inflammatory mediators implicated in acne as potential targets. Immunological and bacterial aspects involved in acne pathogenesis have been considered. In early acne lesions, CD4(+) lymphocytes infiltrate around the follicles [8]. Within the microcomedo, macrophages, CD3(+), and CD4(+) cells are observed along with the activation of vascular adhesion molecules, E-selectin, and integrin [9]. That study confirmed that inflammation precedes hyperkeratinization in early acne lesions [9]. Moreover, C.acnes in the intrafollicular ducts and sebaceous glands in acne stimulate Langerhans cells, infundibular keratinocytes, and sebocytes via Toll-like receptor 2 (TLR2), resulting in the production of interleukin (IL)12, IL-8, IL-6, interferon- $\gamma$ (IFN $\gamma)$, and tumor 
necrosis factor alpha (TNF $\alpha)$ [2, 10]. Subsequently, inflammatory lesions such as papules and pustules are formed. In addition, Th1 cells are predominantly observed in acne follicles [11]. TLR2 expression is also highly pronounced in acne lesions [12]. C. acnes activates activator protein 1 (AP1) and nuclear factor (NF)- $\kappa \mathrm{B}$, resulting in the production of matrix metalloproteinases (MMPs), TNF $\alpha$, IL-1 $\beta$, and IL-8 [13]. Moreover, IL-17, IL-23, and TNF $\alpha$ are highly expressed in acne lesions [14]. TLR2(+) macrophages and IL-17 cells are also present in acne lesions [12]. In the late stages of acne, memory $\mathrm{T}$ cells, plasma cells, and B cells are predominant in atrophic scars $[15,16]$. C. acnes produces lipase, hyaluronidase, and protease, which may break down the follicular walls, giving rise to foreign body granulomas, hypertrophic scars, and keloids.

Although C. acnes is a resident flora in normal skin, C. acnes is closely involved in acne pathogenesis. It has been shown that $C$. acnes biofilms correlate with hyperkeratinization of the infundibulum [17]. C.acnes activates keratinocytes, macrophages, and sebocytes via TLR2, resulting in the production of TNF $\alpha$, IL$1 \beta$, IL-8, and MMP via AP1 and NF- $\mathrm{BB}$ [12]. Subsequently, MMPs promote collagen degradation and fragmentation, and TNF $\alpha$, IL-1 $\beta$, IL8 , and MMP attract neutrophils, resulting in the rupture of hair follicles and extensive inflammation [13]. Peroxisome proliferator-activated receptor (PPAR) is also involved in the inflammatory process in acne $[1,2]$.

\section{PATHOPHYSIOLOGICAL TARGETS FOR ACNE TREATMENT}

\section{Hyperkeratinization}

Acne is focused around the pilosebaceous unit, which includes a wide follicle connected to a multilobulated sebaceous gland and the hair follicle. Hyperkeratinization occurs in the follicular infundibulum, leading to sticky laminated corneocytes and an increased number and size of keratohyaline granules [18]. Filaggrin [19], keratin (K)16, K17, and IL- $1 \alpha$ are expressed in acne lesions $[20,21]$. Most hair follicles in acne lesions are in the telogen and catagen phases [22]. K79 and K75 are decreased in acne (comedo switch) [23]. Retinoid receptors are present in the isthmus [23].

\section{Sebum}

Acne is a disease associated with sebogenesis. Androgen-dependent sebum is produced in sebaceous glands. Within the sebaceous gland, undifferentiated sebocytes differentiate into mature sebocytes with lipid droplets, resulting in sebum discharge in the follicular channel in a holocrine manner.

C. acnes produces lipase, which can convert sebum derived from the sebaceous gland to free fatty acid (FFA), resulting in comedogenesis $[1,2,24]$. In patients with acne, FFA levels are increased and 50\% higher than those found in individuals without acne [24]. FFA induces nodlike receptor 3 (NLRP3) inflammasome and IL$1 \beta$ [24]. Insulin growth factor 1 (IGF-1) also promotes the proliferation and differentiation of sebocytes and IL-1 $\beta$ expression $[24,25]$. Acne severity correlates with the sebum excretion rate [26]. Qualitative analysis has demonstrated that linoleic acid levels are lower in patients with acne [27]. Lipid peroxide influences hyperkeratosis in the sebaceous ducts and induces the pro-inflammatory cytokines involved in acne pathogenesis [28].

Monosaturated free acids are also relevant in acne [29]. Sapienic acid is significantly expressed in acne lesions [30]. Sapienic acid and oleic acid can promote IL- $1 \alpha$ production, which induces hyperkeratinization in the infundibulum and, in turn, comedogenesis. PPAR, acetylcholine, oxidants, and aryl hydrocarbon receptors (AHR) are also increased in acne [1]. Furthermore, androgens are the most important hormones involved in acne pathogenesis. Increased $5 \alpha$-reductase in the sebaceous glands of acne is implicated in its pathogenesis [2]. Local production of androgens in the skin, particularly high expression and hyperresponsiveness of the androgens, may determine the formation of acne lesions [2]. Androgens influence the proliferation and differentiation of sebocytes. Therefore, anti-androgen therapy is 
useful in the management of sebocyte regulation. Corticotropin-releasing hormone (CRH), thyroid hormone, and growth hormone also exert an effect on sebum secretion. Conversely, estrogen decreases sebum production.

\section{Wound Healing}

In general, wound healing is associated with hypertrophic scarring in the general population, including pregnancy, hypertension, and the female sex. The tension in a wound may also influence the development of hypertrophic scarring. Hence, subcutaneous tissue sutures are used in surgery to reduce the tension in the reticular dermis and subsequent hypertrophic scar development.

Although the most important complication in acne is scar formation including atrophic, hypertrophic, and keloid scars, the process of wound healing in acne has not been emphasized until recently. The pathogenesis of acne should consider and clarify the processes involved in wound healing. Future advances in acne therapy should also consider this. Inflammatory papules, pustules, nodules, subcutaneous cysts, and abscesses may develop into erosions and ulcerations, which may lead to atrophic, hypertrophic, and keloid scars. Atrophic scars are classified into three types: icepick scars, rolling scars, and boxcar scars [31]. The formation of atrophic scars involves B cells and macrophages [16]. In acne conglobata, hidradenitis suppurativa (HS; acne inversa), and perifolliculitis capitis abscedens et suffodiens (PCAS), hypertrophic scars and keloids are frequently observed. Scar formation is associated with persistent inflammation and the depth of inflammation.

If scarring involves the reticular dermis under general wound healing conditions, collagen accumulates and a dermal nodule is produced [32]. The intensity and duration of dermal inflammation are dictated by genetic, systemic, local, and lifestyle risk factors [32]. The key local risk factor is the mechanical forces of the acne wound/scar [33]. Mechanical forces strongly influence inflammation-driven pathological scarring.
In hypertrophic scars, the associated clinical features include pregnancy, hypertension, and possibly the female sex. Associations with high circulating concentrations of inflammatory cytokines have also been reported [34]. Genetic causes include single nucleotide polymorphisms [35]. Hypertrophic scarring is associated with innate and adaptive immunity in patients with acne, in which a scar mounts a different innate response when compared to those patients without scarring [15]. Therefore, individual susceptibility may influence the response to inflammation in acne.

The intensity and duration of dermal inflammation influences the production of keloidal collagen around the blood vessels in the reticular dermis [36]. This status is called "keloid" rather than "hypertrophic scars".

\section{Upregulation of Gene Expression}

Transforming growth factor- $\beta$ (TGF $\beta)$, IL-6, MMP, IGF-1, and B cells are found in keloid/ hypertrophic scar tissues [37, 38]. In atrophic scars, TLR4, IL-2, IL-10, tissue inhibitor of metalloprotease 2 (TIMP2), JUN, and TGF $\beta$ are elevated $[39,40]$. Immunological responses are different between patients with and without scarring [15].

\section{UPDATED TARGETED THERAPY BASED ON ACNE PATHOGENESIS}

The target cells and organs involved in acne pathogenesis are the sebaceous glands, infundibular keratinocytes, hair follicles, and endocrinological organs such as the adrenal gland, ovary, and brain.

As previously described, novel targets for acne treatment should impact the major etiological factors, namely, sebogenesis, keratinization, relevant bacteria, inflammation, and mechanisms involved in scarring and wound healing.

In the human skin, microbiomes such as C.acnes, coagulase-negative Staphylococcus (CNS), and fungal species interplay to maintain healthy skin by balancing the homeostatic 
relationships [41]. C.acnes proliferation is believed to be attributable to acne pathogenesis. However, C. acnes proliferation is not related to acne pathogenesis [42]. Instead, it is the $C$. acnes phylotype that is more critically related to acne pathogenesis [42]. In other words, the loss of diversity of the $C$. acnes phylotype is involved in acne pathogenesis [42]. Propionibacterium acnes was renamed Cutibacterium acnes according to biochemical and genomic investigations [43]. Unique genomic elements seem to be associated with acne [41]. Acne-associated C. acnes induces inflammatory cytokines, including high levels of IFN $\gamma$ and IL-17 [41]. In contrast, health-associated C. acnes induces high levels of anti-inflammatory IL-10 [41]. Therefore, the differential functions of the $C$.acnes strains in both host immune responses are related to acne pathogenesis. Microbial dysbiosis can influence innate immunity through PPAR, IFN signaling, TNF activation, IL, TLR, and MMP [41]. Dysbiosis of the skin microbiome, e.g., between C. acnes and Staphylococcus epidermidis, results in acne development [44]. This imbalance may also induce epithelial dysfunction, immune dysregulation, and the overgrowth of pathogenic microbes [41]. Treatments that modify the microbiome other than antibiotics may provide a new generation of ecobiological antiinflammatory treatment for acne [44]. C. acnes is a key player in acne pathogenesis and triggers inflammatory responses through innate immunity, and maintains inflammation by acquired cell-mediated responses through Th1 cells. C. acnes is involved in acne pathogenesis by releasing extracellular enzymes, reactive oxygen, TLR through innate immunity, sebum production, keratin, filaggrin, and IGF, resulting in inflammation and hyperkeratosis [45]. The role of C.acnes in the pathogenesis of acne is described in another review [45].

Acne may also be considered an autoinflammatory disease, as it can be associated with syndromes including acne fulminans (AF); synovitis, acne, pustulosis, hyperostosis, osteitis (SAPHO) syndrome; pyogenic arthritis, pyoderma gangrenosum, acne (PAPA) syndrome; and pyoderma gangrenosum, acne, suppurative hidradenitis (PASH) syndrome. It is also associated with polycystic ovary syndrome (PCOS), metabolic skin diseases with insulin resistance, and endocrinological disorders with excessive androgen production. The inflammasomes are involved in acne pathogenesis. These innate immune system receptors and sensors are related to IL-1 $\beta$, TNF $\alpha$, and Th17 differentiation. IL6 and IL- 8 are also implicated in the pathogenesis of acne. Therefore, studies examining targeted therapies focused on these cytokines are required in the future.

\section{POTENTIAL TARGETS FOR ACNE MANAGEMENT}

\section{Targets for Inflammation}

The mainstays of treatment for inflammatory acne in the acute phase are $\mathrm{BPO}$, adapalene, and antimicrobials. BPO is widely used to treat acne. BPO has diverse mechanisms of action and can act on multifocal sites, such as the cell wall, ribosome, and DNA gyrase in bacteria, resulting in the inhibition of bacterial growth and inflammation in acne [46]. In addition, BPO is comedolytic. Recently, BPO has been shown to inhibit the production of C. acnes biofilms [47]. Antimicrobials have bactericidal or bacteriostatic effects on C. acnes. In contrast, new quinolones, tetracyclines, and macrolides, which inhibit DNA gyrase and protein synthesis, have anti-inflammatory effects. They can inhibit cytokines, such as IL-6 and IL-8 [6].

Other therapies that inhibit inflammation in acne are biological agents. TNF $\alpha \mathrm{Ab}$ is used for HS (acne inversa) [48, 49] and is effective in severe acne, such as acne conglobata (AC) and PCAS (Hoffman) [50, 51]. The Th17 pathway is noteworthy in the inflammatory processes observed in acne-related diseases [14]. IL-17 is a key cytokine that recruits and attracts neutrophil infiltration and can target keratinocytes, endothelial cells, monocytes, and fibroblasts to induce pro-inflammatory mediators $[14,52,53]$. IL-17 Ab can also reduce Th17 inflammation and prevent scar formation [53]. The C.acnes membrane components activate NLRP3 inflammasome in monocytes, resulting in increased secretion of IL-1 $\beta$, which activates Th17 response with production of IL-17 [54]. IL- 
$1 \beta$ antagonists are also effective against acne [54]. Antibodies against IL-12, IL-23, and phosphodiesterase 4 (PDE4) have been shown to be effective against HS [55]. Further large-scale randomized, placebo-controlled clinical trials are needed to examine this approach in acne.

\section{Targets for Hyperkeratinization}

There are many substances that regulate or improve abnormal keratinization in the follicular infundibulum. IL- $1 \alpha$ promotes hyperkeratosis in acne. An IL- $1 \alpha$ antagonist (anakinra) is available for the treatment of HS [56], and IL-1 $\beta$ antagonists are also effective for HS [57].

Adapalene can regulate abnormal keratinization in the infundibulum of acne lesions. A novel fourth-generation topical retinoid (trifarotene) has been developed that specifically targets retinoic acid receptor gamma [58]. This agonist is now licensed for facial and truncal acne with high efficacy, safety, and tolerability [58]. BPO has comedolytic effects. Adapalene and BPO decrease the expression of TLR2 ex vivo [59]. Oral isotretinoin can also inhibit hyperkeratosis in the infundibulum and block TLRs [60].

\section{Targets for Sebogenesis}

Medications to reduce sebum include orally administered isotretinoin in men and women and hormonal therapies in women. To date, there have not been any topical agents that can effectively reduce sebum. A novel topical antiandrogen agent, clascoterone, has just been licensed for patients with acne, aged 9 years and older. It may be the first topical agent that can reduce sebum [61].

Isotretinoin can inhibit sebum production and inhibit the size of the sebaceous glands [3]. Systemic type I 5a-reductase inhibitors did not improve acne in vivo [62]. Topical linoleic acid has shown some efficacy in acne, but there is no significant reduction in sebum [63]. Systemic anti-androgens are effective against acne [7, 64]. Topical anti-androgens are effective against acne [65]. IGF-1 modulators have also been reported as effective treatments for acne [66].
Metformin, an antidiabetic and cancer-protective drug, may be a candidate for acne management by decreasing the expression of mechanistic target of rapamycin complex (mTORM) [30]. Further studies are required to confirm these findings.

\section{Targets for Wound Healing}

In acne, ulceration and erosion may be observed and these contribute to visible scar formation. If inflammation involves the reticular dermis, hypertrophic scars and keloids may develop. Inflammation can also contribute to hyperpigmentation or hypopigmentation. The sequelae of acne are very challenging to treat. Therefore, rapid wound healing is necessary to avoid longterm and permanent visible marks.

Corticosteroid tapes are the first-line therapy in Japan in the acute phase of hypertrophic scar development $[67,68]$. Comparing with corticosteroid injections, tapes can be used locally in the affected area. If conservative therapy does not work, surgery is an option [69]. Following surgery, subcutaneous tissue sutures are important for reducing the tension of the reticular dermis. Surgery and postoperative radiotherapy are considered standard clinical practices for extensive inflammatory keloids [31, 69].

\section{Alternative Therapies}

Ectopeptidase inhibitors, 5-lipoxygenase inhibitors, antisense oligonucleotides, antimicrobial peptides, epigallocatechin-3-gallate in green tea, dietary manipulation such as reducing milk consumption, and gene therapy have all been reported as possible options for the treatment of acne in the future $[1,2]$. Further studies are required to provide robust evidence to support these treatments.

\section{FUTURE TREATMENT FOR ACNE}

The future treatment of acne is summarized in Fig. 1.

Treatment is divided into effective therapies that target different etiological factors. 
The future treatment for acne

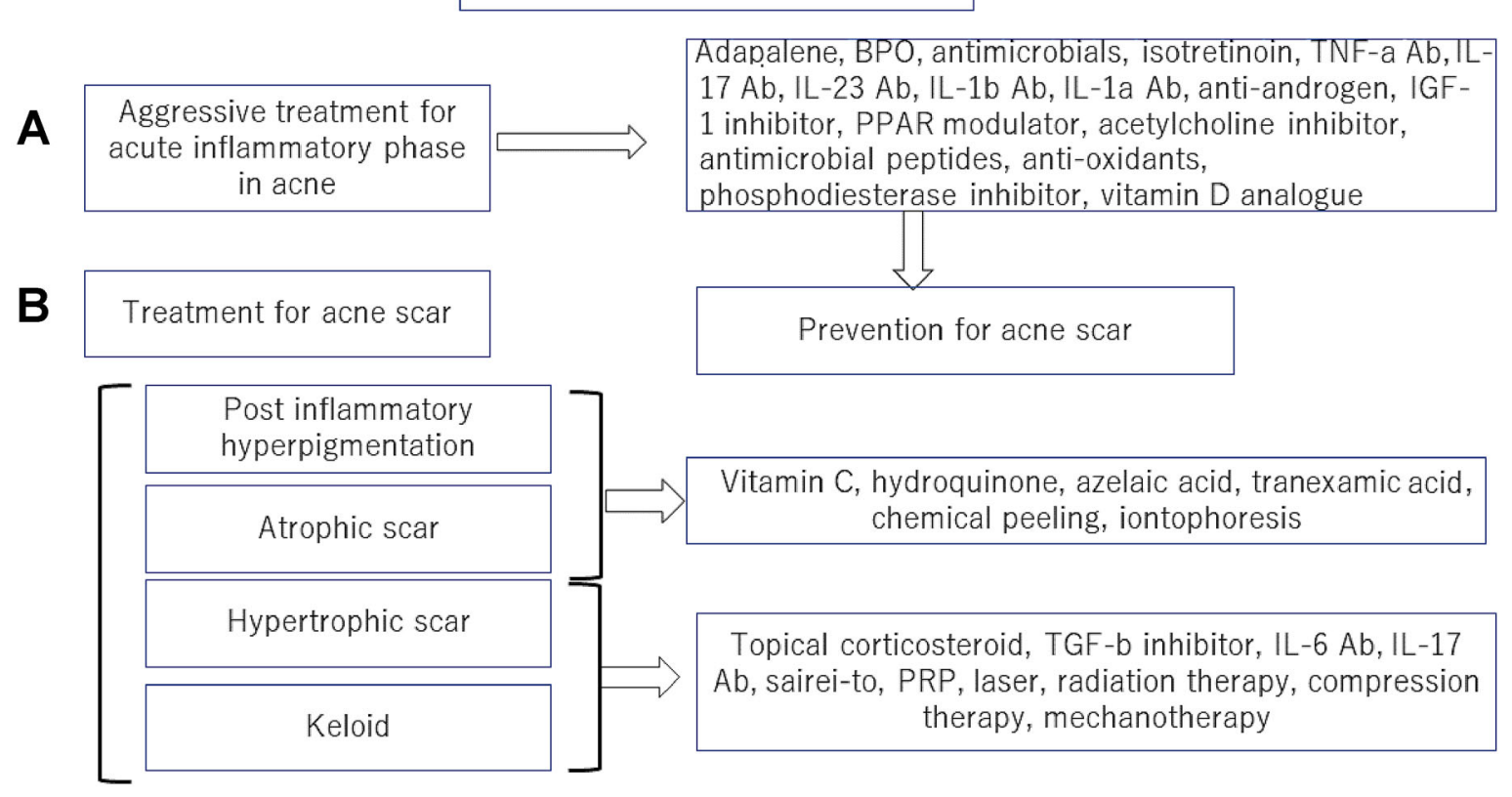

Fig. 1 The future treatment of acne. a First, prevention of acne scars by aggressive treatment during the acute inflammatory phase. $\mathbf{b}$ Treatment for acne scars (post-inflammatory hyperpigmentation, hypertrophic scars, and keloids)

Treatment must aim to treat the acne and avoid any sequelae. Possible treatment modalities for clinical sequelae that may arise should also be considered.

\section{Treatment for Acne}

Early effective therapy is important to avoid acne sequelae. Prevention of acne-induced scarring should be avoided by adopting aggressive treatment in the acute inflammatory phase.

There is robust evidence in acne guidelines to support the use of medications such as adapalene, BPO, antimicrobials, and isotretinoin.

Other optional treatments that have the potential to offer effective treatment in the future include TNF $\alpha$, IL-17, IL-23, IL-1 $\beta$, IL-1 $\alpha$, anti-androgen, IGF-1, PPAR, acetylcholine, antimicrobials, antioxidants, phosphodiesterase, and vitamin D analogues.

\section{Possible Treatments for Acne Sequelae}

\section{Post-inflammatory Hyperpigmentation}

Vitamin C, hydroquinone, azelaic acid, tranexamic acid, chemical peeling, and iontophoresis can be used.

\section{Hypertrophic Scar and Keloid}

Topical corticosteroids injection, vitamin C, hydroquinone, azelaic acid, tranexamic acid, chemical peeling, iontophoresis, TGF $\beta$ inhibitor, IL-6 Ab, IL-17 Ab, saireito (a Japanese herb), platelet-rich plasma (PRP), laser, radiation therapy, compression therapy, and mechanotherapy can be used.

\section{CONCLUSION}

In this review, we summarize updated treatments facilitating potential novel approaches in acne treatment including immunology and wound healing. In particular, biological treatments targeting IL-1 $\beta$, IL-17, IL-23, and TNF $\alpha$ 
could provide novel approaches for treating severe acne and related disorders. In addition, biological antibodies targeting TGF $\beta$, IL-6, MMP, IGF-1, and B cells may be a potential strategy for the prevention and treatment of this type of scar in the future. Future treatment for acne should embrace approaches that target the main etiological factors of acne.

\section{ACKNOWLEDGEMENTS}

Funding. No sponsorship was received for this publication of this article.

Authorship. All the named authors meet the International Committee of Medical Journal Editors (ICMJE) criteria for authorship for this article, take responsibility for the integrity of the work as a whole, and have given their approval for this version to be published.

Medical Writing and Editorial Assistance. We thank Editage company for linguistic assistance in editing the English text of the revised manuscript. The fee was funded by authors.

Disclosures. Ichiro Kurokawa and Rei Ogawa have nothing to disclose. Alison M Layton has recieved unrestricted educational talks/ research grants or consultancies over the last 5 years from the following companies Galderma, GSK, La Roche Posay, Leo, L'Oreal, Novartis, Origimm, Proctor and Gamble.

Compliance with Ethics Guideline. This article is based on previously conducted studies and does not contain any new studies with human participants or animals performed by any of the authors.

Data Availability. Data sharing is not applicable to this article as no datasets were generated or analyzed during the current study.

Open Access. This article is licensed under a Creative Commons Attribution-NonCommercial 4.0 International License, which permits any non-commercial use, sharing, adaptation, distribution and reproduction in any medium or format, as long as you give appropriate credit to the original author(s) and the source, provide a link to the Creative Commons licence, and indicate if changes were made. The images or other third party material in this article are included in the article's Creative Commons licence, unless indicated otherwise in a credit line to the material. If material is not included in the article's Creative Commons licence and your intended use is not permitted by statutory regulation or exceeds the permitted use, you will need to obtain permission directly from the copyright holder. To view a copy of this licence, visit http://creativecommons.org/licenses/by$\mathrm{nc} / 4.0 /$.

\section{REFERENCES}

1. Kurokawa I, Nakase K. Recent advances in understanding and managing acne. F1000Res. 2020;9: 792.

2. Kurokawa I, Kurokawa I, Danby FW, Ju Q. New developments in our understanding of acne pathogenesis and treatment. Exp Dermatol. 2009;18:821-32.

3. Peck GL, Olsen TG, Butkus D, et al. Isotretinoin versus placebo in the treatment of cystic acne. A randomized double-blind study. J Am Acad Dermatol. 1982;6(4 Pt 2 Suppl):735-45.

4. Dreno B, Gollnick HP, Kang S, et al. Understanding innate immunity and inflammation in acne: implications for management. J Eur Acad Dermatol Venereol. 2015;29(Suppl 4):3-11.

5. Jansen T, Plewig G. Advances and perspectives in acne therapy. Eur J Med Res. 1997;28(2):321-34.

6. Layton AM, Eady EA. Benzoyl peroxide and adapalene fixed combination: a novel agent for acne. Br J Dermatol. 2009;161:971-6.

7. Bettoli V, Zauli S, Virgili A. Is hormonal treatment still an option in acne today? $\mathrm{Br} \mathrm{J}$ Dermatol. 2015;172(Suppl 1):37-46.

8. Layton A, Morris C, Cunliffe WJ, Ingham E. Immunohistochemical investigation of evolving inflammation in lesions of acne vulgaris. Exp Dermatol. 1998;7:191-7. 
9. Jeremy AH, Holland DB, Roberts SG, Thomson KF, Cunliffe WJ. Inflammatory events are involved in acne lesion initiation. J Invest Dermatol. 2003;121: 20-7.

10. Sugisaki H, Yamanaka K, Kakeda M, et al. Increased interferon-gamma, interleukin-12p40 and IL-8 production in Propionibacterium acnes-treated peripheral blood mononuclear cells from patient with acne vulgaris: host response but not bacterial species is the determinant factor of the disease. J Dermatol Sci. 2009;55:47-52.

11. Mouser PE, Baker BS, Seaton ED, Chu AC. Propionibacterium acnes-reactive T helper-1 cells in the skin of patients with acne vulgaris. J Invest Dermatol. 2003;121:1226-8.

12. Kim J. Review of the innate immune response in acne vulgaris: activation of Toll-like receptor 2 in acne triggers inflammatory cytokine responses. Dermatology. 2005;211:193-8.

13. Kang Am J, Pathol Kang S, Cho S, et al. Inflammation and extracellular matrix degradation mediated by activated transcription factors nuclear factor- $\kappa \mathrm{B}$ and activator protein-1 in inflammatory acne lesions in vivo. Am J Pathol. 2005;166:1691-9.

14. Kelhälä HL, Palatsi R, Fyhrquist N, et al. IL-17/Th17 pathway is activated in acne lesions. PLoS ONE. 2014;25(9):e105238.

15. Holland DB, Jeremy AH, Roberts SG, Seukeran DC, Layton AM, Cunliffe WJ. Inflammation in acne scarring: a comparison of the responses in lesions from patients prone and not prone to scar. Br J Dermatol. 2004;150:72-81.

16. Carlavan B, Carlavan I, Bertino B, Rivier M. Atrophic scar formation in patients with acne involves long-acting immune responses with plasma cells and alteration of sebaceous glands. Br J Dermatol. 2018;179:906-17.

17. Burghart Burkhart CG, Burkhart CN. Expanding the microcomedone theory and acne therapeutics: Propionibacterium acnes biofilm produces biological glue that holds corneocytes together to form plug. J Am Acad Dermatol. 2007;57:722-4.

18. Knutson DD. Ultrastructural observations in acne vulgaris: the normal sebaceous follicle and acne lesions. J Invest Dermatol. 1974;62:288-307.

19. Kurokawa I, Mayer-da-Silva A, Gollnick H, Orfanos CE. Monoclonal antibody labeling for cytokeratins and filaggrin in the human pilosebaceous unit of normal, seborrhoeic and acne skin. J Invest Dermatol. 1988;91:566-71.
20. Hughes BR, Morris C, Cunliffe WJ, Leigh IM. Keratin expression in pilosebaceous epithelia in truncal skin of acne patients. Br J Dermatol. 1996;134: 247-56.

21. Guy R, Green MR, Kealey T. Modeling acne in vitro. J Invest Dermatol. 1996;106:176-82.

22. Van Scotte EJ, Maccardle RC. Keratinization of the duct of the sebaceous gland and growth cycle of the hair follicle in the histogenesis of acne in human skin. J Invest Dermatol. 1956;27:405-29.

23. Fantao F, von Engelbrechten M, Seilaz C, Sorg O, Saurat JH. Microcomedones in non-lesional acne prone skin: new orientations on comedogenesis and its prevention. J Eur Acad Dermatol Venereol. 2020;34:357-64.

24. Plewig G, et al. Acne pathogenesis. In: Plewig G, Melnik B, Chen WC, editors. Plewig and Kligman's acne and rosacea. 4th ed. Berlin: Springer; 2019. p. $45-51$.

25. Picardo M, Eichenfeld L, Tan J. Acne and rosacea. Dermatol Ther (Heidelb). 2017;7(suppl 1):S43-s52.

26. Cotterill JA, Cunliffe WJ, Williamson B. Severity of acne and sebum excretion rate. Br J Dermatol. 1971;85:93-4.

27. Downing DT, Stewart ME, Wertz PW, Strauss JS. Essential fatty acids and acne. J Am Acad Dermatol. 1986;14(2 Pt 1):221-5.

28. Ottaviani M, Camera E, Picardo M. Lipid mediators. Mediators Inflamm. 2010;2010:858176. https://doi. org/10.1155/2010/858176.

29. Katsuta Y, Iida T, Inomata S, Denda M. Unsaturated fatty acids induce calcium influx into keratinocytes and cause abnormal differentiation of epidermis. J Invest Dermatol. 2005;124:1008-13.

30. Melnik B. Acne vulgaris: the metabolic syndrome of the pilosebaceous follicle. Clin Dermatol. 2018;36: 29-40.

31. Jacob CI, Dover JS, Kaminer MS. Acne scarring: a classification system and review of treatment options. J Am Acad Dermatol. 2001;45:109-17.

32. Ogawa R. Keloid and hypertrophic scars are the result of chronic inflammation in the reticular dermis. Int J Mol Sci. 2017;18:606.

33. Harn HI, Ogawa R, Hsu CK, Hughes MW, Tang MJ, Chuong CM. The tension biology of wound healing. Exp Dermatol. 2019;28:464-71. 
34. Noishiki C, Hayasaka Y, Ogawa R. Sex differences in keloidogenesis: an analysis of 1659 keloid patients in Japan. Dermatol Ther (Heidelb). 2019;9:747-54.

35. Ogawa R, Watanabe A, Naing BT, et al. Associations between keloid severity and single-nucleotide polymorphisms: importance of rs8032158 as a biomarker of keloid severity. J Invest Dermatol. 2014;134:2041-3.

36. Matsumoto NM, Peng WX, Aoki M, et al. Histological analysis of hyalinised keloidal collagen formation in earlobe keloids over time: collagen hyalinisation starts in the perivascular area. Int Wound J. 2017;14:1088-93.

37. Suh DH, Kwon HH. What's new in the physiopathology of acne? Br J Dermatol. 2015;172(Suppl 1):13-9.

38. Yang JH, Yon JY, Moon J, Min S, Kwon HH, Suh DH. Expression of inflammatory and fibrogenetic markers in acne hypertrophic scar formation: focusing on role of TGF- $\beta$ and IGF-1R. Arch Dermatol Res. 2018;310:665-73.

39. Saint-Jean M, Khammari A, Jasson F, Nguyen JM, Dréno B. Different cutaneous innate immunity profiles in acne patients with and without atrophic scars. Eur J Dermatol. 2016;26:68-74.

40. Moon J, Yoon JY, Yang JH, Kwon HH, Min S, Suh DH. Atrophic acne scar: a process from altered metabolism of elastic fibres and collagen fibres based on transforming growth factor- $\beta 1$ signalling. Br J Dermatol. 2019;181:1226-37.

41. Ramasamy S, Barnard E, Dawson TL Jr, Li H. The role of the skin microbiota in acne pathophysiology. Br J Dermatol. 2019;181:691-9.

42. Dréno B, Pécastaings S, Corvec S, Veraldi S, Khammari A, Roques C. Cutibacterium acnes (Propionibacterium acnes) and acne vulgaris: a brief look at the latest updates. J Eur Acad Dermatol Venereol. 2018;32(Suppl 2):5-14.

43. Scholz CFP, Kilian M. The natural history of cutaneous propionibacteria, and reclassification of selected species within the genus Propionibacterium to the proposed novel genera Acidipropionibacterium gen. nov., Cutibacterium gen. nov. and Pseudopropionibacterium gen. nov. Int J Syst Evol Microbiol. 2016;66:4422-32.

44. Dréno B, Dagnelie MA, Khammari A, Corvec S. The skin microbiome: a new actor in inflammatory acne. Am J Clin Dermatol. 2020;21(Suppl 1):18-24.

45. Beylot C, Auffret N, Poli F, et al. Propionibacterium acnes: an update on its role in the pathogenesis of acne. J Eur Acad Dermatol Venereol. 2014;28: 271-8.

46. Burkhart CN, Burkhart CG. Microbiology's principle of biofilms as a major factor in the pathogenesis of acne vulgaris. Int J Dermatol. 2003;42:925-7.

47. Tabara K, Tamura R, Nakamura A, et al. Anti-inflammatory effects of ozenoxacin, a topical quinolone antimicrobial agent. J Antibiot (Tokyo). 2020;73:247-54.

48. Zouboulis CC, Desai N, Emtestam L, et al. European $\mathrm{S} 1$ guideline for the treatment of hidradenitis suppurativa/acne inversa. J Eur Acad Dermatol Venereol. 2015;29:619-44.

49. Morita A, Takahashi H, Ozawa K, et al. Twenty-fourweek interim analysis from a phase 3 open-label trial of adalimumab in Japanese patients with moderate to severe hidradenitis suppurativa. J Dermatol. 2019;46:745-51.

50. Yiu ZZ, Madan V, Griffiths CE. Acne conglobata and adalimumab: use of tumour necrosis factor- $\alpha$ antagonists in treatment-resistant acne conglobata, and review of the literature. Clin Exp Dermatol. 2015;40:383-6.

51. Takahashi T, Yamasaki K, Terui H, et al. Perifolliculitis capitis abscedens et suffodiens treatment with tumor necrosis factor inhibitors: a case report and review of published cases. J Dermatol. 2019;46: 802-7.

52. Kistowska M, Meier B, Proust T, et al. Propionibacterium acnes promotes Th17 and Th17/Th1 responses in acne patients. J Invest Dermatol. 2015;135:110-8.

53. Agak GW, Qin M, Nobe J, et al. Propionibacterium acnes induces an IL-17 response in acne vulgaris that is regulated by vitamin A and vitamin D. J Invest Dermatol. 2014;134:366-73.

54. Thiboutot DM, Layton AM, Eady AE. IL-17: a key player in the $P$. acnes inflammatory cascade? J Invest Dermatol. 2014;134:307-10.

55. Vossen ARJV, van Doorn MBA, van der Zee HH, Prens EP. Apremilast for moderate hidradenitis suppurativa: results of a randomized controlled trial. J Am Acad Dermatol. 2019;80:80-8.

56. Maarouf M, Clark AK, Lee DE, Shi VY. Targeted treatments for hidradenitis suppurativa: a review of the current literature and ongoing clinical trials. J Dermatolog Treat. 2018;29:441-9.

57. Dinarello CA, van der Meer JW. Treating inflammation by blocking interleukin-1 in humans. Semin Immunol. 2013;25:469-84. 
58. Tan J, Miklas M. A novel topical retinoid for acne: trifarotene $50 \mu \mathrm{g} / \mathrm{g}$ Cream. Skin Therapy Lett. 2020;25:1-2.

59. Zuliani T, Khammari A, Chaussy H, Knol AC, Dréno B. Ex vivo demonstration of a synergistic effect of adapalene and benzoyl peroxide on inflammatory acne lesions. Exp Dermatol. 2011;20:850-3.

60. Dispenza MC, Wolpert EB, Gilliland KL, et al. Systemic isotretinoin therapy normalizes exaggerated TLR-2-mediated innate immune responses in acne patients. J Invest Dermatol. 2012;132:2198-205.

61. Hebert A, Thiboutot D, Stein Gold L, et al. Efficacy and safety of topical clascoterone cream, $1 \%$, for treatment in patients with facial acne: two phase 3 randomized clinical trials. JAMA Dermatol. 2020;156:621-30.

62. Leyden J, Bergfeld W, Drake L, et al. A systemic type I 5 alpha-reductase inhibitor is ineffective in the treatment of acne vulgaris. J Am Acad Dermatol. 2004;50:443-7.

63. Letawe C, Boone M, Piérard GE. Digital image analysis of the effect of topically applied linoleic acid on acne microcomedones. Clin Exp Dermatol. 1998;23:56-8.

64. Zouboulis CC, Bettoli V. Management of severe acne. Br J Dermatol. 2015;172(Suppl 1):27-33.

65. Marks DH, Prasad S, De Souza B, Burns LJ, Senna MM. Topical antiandrogen therapies for androgenetic alopecia and acne vulgaris. Am J Clin Dermatol. 2020;21:245-54.

66. Cong TX, Hao D, Wen X, Li XH, He G, Jiang X. From pathogenesis of acne vulgaris to anti-acne agents. Arch Dermatol Res. 2019;311:337-49.

67. Goutos I, Ogawa R. Steroid tape: a promising adjunct to scar management. Scars Burn Heal. 2017;3:2059513117690937.

68. Ogawa R, Akita S, Akaishi S, et al. Diagnosis and treatment of keloids and hypertrophic scars-Japan Scar Workshop consensus document. Burns Trauma. 2018;2019:7-39.

69. Ogawa R, Tosa M, Dohi T, Akaishi S, Kuribayashi S. Surgical excision and postoperative radiotherapy for keloids. Scars Burn Heal. 2019;5:1-11. 